\title{
PROLONGATION OF ORAL PHASE FOR INITIAL SWALLOW OF SOLID FOOD IS ASSOCIATED WITH ORAL DIADOCHOKINESIS DETERIORATION IN NURSING HOME RESIDENTS IN JAPAN: A CROSS-SECTIONAL STUDY
}

\author{
M. Shimosaka ${ }^{1}$, W. Fujii ${ }^{1}$, Y. Kakinoki², S. Akifusa ${ }^{1}$
}

\begin{abstract}
Background: Prolongation of bolus forming complicates ingestion, in particular in older adults. Objectives: The purpose of this study is to examine which oral functions are associated with prolongation of the oral phase of forming a bolus until swallowing in older adults. Design: Cross-sectional study. Setting: three nursing homes in Kitakyushu, Japan from August 2017 to October 2018. Participants: 39 adults >60-years. Measurements: Number of functional teeth, chewing ability, swallowing ability, tongue and cheek pressure, saliva flow rate, oral diadochokinesis, global cognitive function, and body mass index, were examined. Time of oral phase until the first swallowing of solid food was measured as the outcome of the study using video, and audio recording of the swallowing sound by a throat microphone, with the cutoff point designated at $30 \mathrm{~s}$. Based on the oral phase, participants were divided in two groups: normal and prolonged. Results: The 39 enrolled participants had a median age of 87 years, $17.3 \%$ were men, and $48.7 \%$ had prolonged oral phase. In the prolonged group, the swallowing ability, saliva flow rate, tongue and cheek pressure, and oral diadochokinesis were significantly lower than in the normal group. Binomial logistic regression analysis revealed that oral phase prolongation was associated with oral diadochokinesis (odds ratio $0.81,95 \%$ confidence interval $0.67-0.98$ ) after adjusting for potential covariates. Conclusion: Oral diadochokinesis deterioration is significantly associated with oral phase prolongation for initial swallowing of solid food in older adults.
\end{abstract}

Key words: Mastication, oral diadochokinesis, prolongation of oral phase, swallowing.

\section{Introduction}

Oral function depends on various factors, including the number of natural teeth, chewing ability, oral diadochokinesis (ODK), tongue pressure, and swallowing ability, which are all indispensable for maintaining a good nutritional status. A decline in oral function frequently restrains dietary intake in older adults (1, 2 ). Nursing protocols that include oral care assistance contribute to reducing weight loss and decline in nutritional status, as assessed by the Mini-Nutritional Assessment (3). It is frequently observed that the time from mouthing food to the initial swallowing tends to be prolonged in older adults (4). However, the cause of such prolongation remains unclear. It has been reported that the number of masticatory cycles and oral phase duration increase with age, leading to prolongation of

1. School of Oral Health Sciences, Faculty of Dentistry, Kyushu Dental University, Kitakyushu, Japan; 2. Division of Special Needs and Geriatric Dentistry, Faculty of Dentistry, Kyushu Dental University, Kitakyushu, Japan.

Corresponding Author: Sumio Akifusa, School of Oral Health Sciences, Faculty of Dentistry, Kyushu Dental University, 2-6-1, Manazuru, Kokurakita-ku, Kitakyushu 803-8580, Japan. E-mail: r11akifusa@fa.kyu-dent.ac.jp forming a bolus (5). Therefore, the aim of the present study was to examine which oral factors are associated with prolongation of oral phase without swallowing in older adults.

\section{Methods}

The reporting of this study follows the STrengthening the Reporting of OBservational studies in Epidemiolog (STROBE) guidelines for the analysis of observational data.

\section{Study setting and participants}

This cross-sectional study was carried out at three nursing care homes for older adults in Kitakyushu, Japan. The study commenced in August 2017, with enrollment ending in October 2018. Forty-two residents of nursing homes, aged $>60$-years-old, were included in this study. Residents with psychiatric disorders, systemic inflammation, head and neck tumors, Sjögren syndrome, and severe medical history of digestive system disease were excluded from this study. Thus, a total of 39 
participants were included in the final analysis. Informed consent was obtained from all participants or their legal guardians. This study was conducted with the approval of the ethics review board of Kyushu Dental University (No.: 16-102). The research was carried out in accordance with the Declaration of Helsinki.

\section{Data collection}

Information regarding age, sex, body mass index (BMI) in $\mathrm{kg} / \mathrm{m}^{2}$, medical history, motor palsy, and choking was obtained from clinical records. Global cognitive function, measured using the 30-question Mini-Mental State Examination (MMSE) (6), was collected from medical records. A trained dental hygienist carried out all measurements of oral function. In the present study, a functional tooth was defined as a tooth having occlusal function without severe periodontitis or loss of the crown.

\section{Swallowing function assessment}

The participants' swallowing ability was assessed using the repetitive saliva swallowing test $(\operatorname{RSST})(7,8)$. While seated, participants were asked to swallow their saliva as many times as possible in $30 \mathrm{~s}$. The number of swallows were counted by the examiner through monitoring of larynx palpation, which gave a ratio scale measurement.

\section{Tongue and cheek pressure measurement}

Tongue and cheek pressure were evaluated using a specified tongue pressure measurement device (JMS Co, TPM-01, Japan). TPM-01 is a handheld manometer consisting of a small balloon-type disposable oral probe. At zero calibration, the probe is inflated with air at a pressure of $19.6 \mathrm{kPa}$ (9). The measurement was performed with participants sitting in a relaxed position, and participants were asked to squash, with as much force as possible for 7 seconds, the balloon probe interposed between their tongue and palate to measure tongue pressure or between the buccal mucosa and buccal aspect of the first molar to measure cheek pressure. For tongue pressure, the measurement was performed 3 times, and the maximum value was used. For cheek pressure, the pressure of each cheek was measured, and the maximum value was used.

\section{ODK measurement}

ODK was defined as the motor skill and the rate of movement of the lips and tongue, according to a previous study (10). The ODK test was used to evaluate the number of fastest repetitive articulations of / pa/, / ta/ and / ka/ within a period of $5 \mathrm{~s}$ and without taking a breath, using the pen-tapping method, and then to calculate the pronunciations per second. These articulations were used to evaluate the movement of lips, apex of the tongue, and posterior region of the tongue, respectively (10). The total ODK test score was calculated by summing scores in all elements.

\section{Saliva flow volume}

Saliva flow rate was measured as per a previous study with some slight modifications (11). Participants were instructed not to smoke, eat, and drink before the assessment. A dry medical gauze was weighed in a laboratory dish before participants were instructed to swallow all the saliva present in the oral cavity and then chew on the gauze for $2 \mathrm{~min}$. The gauze was then weighed again, and the difference in the two weight measurements was defined as the amount of secreted saliva.

\section{Functional tooth units (FTUs)}

We calculated the FTUs based on the clinical examination of the participants (12). FTUs were defined as pairs of opposing posterior natural teeth or prostheses (fixed or removable dentures), excluding carious teeth with extensive coronal destruction. In this study, two opposing premolars were defined as one FTU, and two opposing molars were defined as two FTUs. Therefore, the maximum total FTU score was 12.

\section{Chewing ability}

Chewing ability was assessed using a visual scoring method (13). After being given a piece of gummy jelly (UHA Mikakuto Co., Osaka, Japan), which is a standardized testing food, participants were instructed to chew the piece 30 times on their preferred chewing side and then expectorate the comminuted particles. Masticatory performance was scored using a scale of 0 to 9 by comparing images of visual samples of the particles, with a smaller score indicating better chewing ability.

\section{Oral phase and residual amount of food bolus}

To assess oral phase until initial swallowing of solid food and the residual amount of the food bolus, participants were instructed to eat $8 \mathrm{~g}$ of Processlead ${ }^{\circledR}$ (Otsuka Pharmaceutical Factory, Inc., Japan) in a normal manner. Processlead ${ }^{\circledR}$ is a novel chew-swallow managing food, based on a process model (14). The amount of Processlead ${ }^{\circledR}$ was standardized at $8 \mathrm{~g}$ to avoid bolting without chewing (15). The oral phase was measured from food intake to the first swallowing. Food intake was video recorded, and the swallowing sound was recorded using a throat microphone (SH-12jk, NANZU ELECTRIC Co., Ltd., Japan), attached to the neck. Previously, we 
Table 1

Characteristics of participants

\begin{tabular}{|c|c|c|c|c|}
\hline Variables & Overall $(n=39)$ & Normal group $(n=19)$ & Prolonged group $(n=20)$ & p-value \\
\hline Age, years (min-max) & $87(63-104)$ & 87 (67-98) & $86(63-104)$ & 0.989 \\
\hline Men, n (\%) & $9(17.3)$ & $7(36.8)$ & $2(10.0)$ & 0.053 \\
\hline \multicolumn{5}{|l|}{ Nutritional status } \\
\hline BMI, kg/m2 (min-max) & $19.9(13-30)$ & $20.4(14-25)$ & $19.4(13-30)$ & 0.607 \\
\hline MMSE, m (min-max) & $7(2-14)$ & $15.5(0-29)$ & $6.0(2-11)$ & 0.017 \\
\hline Number of functional teeth, $\mathrm{m}$ (min-max) & $28(0-28)$ & $28(15-28)$ & $27.5(0-28)$ & 0.309 \\
\hline FTU, m (min-max) & $12(0-12)$ & $12(4-12)$ & $12(0-12)$ & 0.365 \\
\hline Chewing ability m (min-max) & $0(0-7)$ & $0(0-7)$ & $0(0-4)$ & 0.513 \\
\hline Chewing time, s m (min-max) & $30(9-134)$ & $24(9-29)$ & $43(30-134)$ & $<0.001$ \\
\hline RSST, m (min-max) & $1(0-7)$ & $2(1-7)$ & $0(0-4)$ & 0.001 \\
\hline Saliva flow, $\mathrm{g} / \mathrm{min}$ (min-max) & $0.9(0-2)$ & $1.2(0.5-2)$ & $0.6(0-2)$ & 0.046 \\
\hline Tongue pressure, $\mathrm{kPa}$ (min-max) & $12.8(0-35)$ & $15.1(3-35)$ & $8.3(0-24)$ & 0.041 \\
\hline Cheek pressure, $\mathrm{kPa}$ (min-max) & $7.5(0-22)$ & $10.5(0-21)$ & $6.2(0-22)$ & 0.044 \\
\hline \multicolumn{5}{|l|}{ Oral diadochokinesis, s-1 } \\
\hline /pa/, m (min-max) & $3.1(0-7)$ & $4.0(0-6)$ & $1.3(0-7)$ & 0.001 \\
\hline$/ \mathrm{ta} /, \mathrm{m}$ (min-max) & $3.2(0-7)$ & $4.3(0-7)$ & $1.2(0-6)$ & $<0.001$ \\
\hline$/ \mathrm{ka} /, \mathrm{m}(\min -\max )$ & $2.5(0-8)$ & $4.4(0-8)$ & $1.3(0-6)$ & 0.002 \\
\hline total, m (min-max) & $9.0(0-21.8)$ & $13.4(0-21.8)$ & $4.2(0-18.2)$ & $<0.001$ \\
\hline \multicolumn{5}{|l|}{ Residual food bolus } \\
\hline $1-2, \mathrm{n}(\%)$ & $25(64.1)$ & $16(84.2)$ & $9(45.0)$ & 0.011 \\
\hline $3-5, \mathrm{n}(\%)$ & $14(35.9)$ & $3(15.8)$ & $11(55.0)$ & \\
\hline Risk of choke, n (\%) & $14(35.6)$ & $4(21.1)$ & $10(50.0)$ & 0.096 \\
\hline Paralysis, n (\%) & $22(56.4)$ & $8(42.1)$ & $14(70.0)$ & 0.111 \\
\hline
\end{tabular}

demonstrated that the average oral phase is about $25 \mathrm{~s}$ in individuals $>70$-years-old (4). Considering that the average age of participants in this study was over 80 years, we designated participants with an oral phase of 30 -s and above in the "prolonged" group and those with $<30 \mathrm{~s}$ in the "normal" group. The residual amount of the food bolus was measured after the first swallowing, by collecting it with a disposable oral care sponge swab and evaluating it in 4 grades: 1) no residual bolus, 2) crumbs, 3 ) less than half of initial bolus, and 4) over half.

\section{Outcome measure}

The outcome measure of this study was the duration of the oral phase until the first swallowing of solid food.

\section{Statistical analysis}

Continuous variables are expressed as the median (minimum to maximum). The Chi-square test and Mann-
Whitney U-test were used to examine differences between the prolonged and normal groups. The correlation between oral functions and oral phase was analyzed using Spearman's rank correlation coefficient. In binomial logistic regression analysis, time of oral phase was included as a dichotomized variable (normal group and prolonged group). Three models were carried out in the binomial logistic regression analysis. Model 1 was the unadjusted model. Model 2 was adjusted for sex and age. Model 3 was adjusted for: sex, age, global cognitive function, swallowing ability, tongue pressure, and cheek pressure. All analyses were carried out using SPSS statistical software version 22 (SPSS, Chicago, IL, USA), and the level of significance was set at $5 \%$ in all cases.

\section{Results}

The participants' characteristics are shown in Table 1. The time of oral phase in the prolonged group [43 (30-134)] was significantly longer than that of the normal 
Table 2

Correlation between time of oral phase and oral functions

\begin{tabular}{|c|c|c|c|c|c|c|c|c|c|c|c|}
\hline & Variables & 1 & 2 & 3 & 4 & 5 & 6 & 7 & 8 & 9 & 10 \\
\hline 1 & $/ \mathrm{pa} /, \mathrm{s}-1$ & 1 & & & & & & & & & \\
\hline 2 & $/ \mathrm{ta} /, \mathrm{s}-1$ & $0.945^{* *}$ & 1 & & & & & & & & \\
\hline 3 & / ka/, s-1 & $0.955^{* *}$ & $0.943^{* *}$ & 1 & & & & & & & \\
\hline 4 & Functional teeth & 0.14 & 0.12 & 0.11 & 1 & & & & & & \\
\hline 5 & FTU & 0.07 & 0.06 & 0.07 & $0.906^{* *}$ & 1 & & & & & \\
\hline 6 & RSST, 30 s-1 & $0.576^{* *}$ & $0.587^{* *}$ & $0.586^{* *}$ & 0.17 & 0.09 & 1 & & & & \\
\hline 7 & Saliva flow, $\mathrm{g} / \mathrm{min}$ & -0.05 & -0.05 & -0.06 & 0.28 & $0.331^{*}$ & 0.17 & 1 & & & \\
\hline 8 & Tongue pressure, $\mathrm{kPa}$ & $0.415^{* *}$ & $0.455^{* *}$ & $0.449^{* *}$ & -0.03 & -0.11 & $0.691^{* *}$ & 0.08 & 1 & & \\
\hline 9 & Cheek pressure, $\mathrm{kPa}$ & $0.470^{* *}$ & $0.501^{* *}$ & $0.481^{* *}$ & 0.17 & 0.18 & $0.652^{* *}$ & 0.23 & $0.704^{* *}$ & 1 & \\
\hline 10 & Oral phase, s & $-0.544^{* *}$ & $-0.561^{* *}$ & $-0.459^{* *}$ & -0.10 & -0.09 & $-0.549^{* *}$ & $-0.418^{*}$ & $-0.345^{*}$ & -0.30 & 1 \\
\hline
\end{tabular}

*: $\mathrm{p}<0.05$; ** $\mathrm{p}<0.01$; FTU: functional tooth unit, RSST: repetitive saliva swallowing test.

Table 3

Binomial logistic regression analysis showing the associations of oral functions with oral phase prolongation

\begin{tabular}{lllcccc}
\hline & \multicolumn{2}{c}{ Model 1 } & \multicolumn{2}{c}{ Model 2 } & \multicolumn{2}{c}{ Model 3 } \\
Variables & & & OR (95\% CI) & p-value & OR (95\% CI) & p-value \\
\hline Total ODK & $0.80(0.70-0.93)$ & 0.003 & $0.80(0.68-0.93)$ & 0.003 & $0.81(0.67-0.98)$ & 0.028 \\
Swallowing function & $0.43(0.23-0.81)$ & 0.008 & $0.45(0.24-0.83)$ & 0.011 & $0.52(0.22-1.21)$ & 0.130 \\
Saliva flow & $0.57(0.30-1.09)$ & 0.091 & $0.49(0.23-1.05)$ & 0.066 & $0.19(0.03-1.02)$ & 0.053 \\
Tongue pressure & $0.92(0.85-1.00)$ & 0.046 & $0.92(0.84-1.01)$ & 0.074 & $1.02(0.87-1.20)$ & 0.822 \\
Cheek pressure & $0.90(0.81-1.01)$ & 0.064 & $0.89(0.78-1.00)$ & 0.057 & $1.01(0.80-1.29)$ & 0.916 \\
\hline
\end{tabular}

OR: odds ratio, CI: confidence interval for odds ratio, ODK: oral diadochokinesis; Total sample size included in the analysis: 39 ; Model 1, unadjusted; Model 2, adjusted by sex and age; Model 3, adjusted by sex, age, global cognitive function, total ODK, swallowing ability, saliva flow, tongue pressure, and cheek pressure.

group [24 (9-29)]. There were no significant differences in age, sex, BMI, number of functional teeth, FTUs, and chewing ability between the prolonged and normal groups. Global cognitive function, as assessed by the MMSE, was significantly lower in the prolonged group when compared to the normal group. Oral functions [e.g., swallowing ability assessed by the RSST, saliva flow rate, tongue and cheek pressure, ODK] were significantly lower in the prolonged group than in the normal group. The participants were divided into two groups according to the amount of residual food, i.e., those with scores of 1-2 were classified as the no residual food group and those with scores of 3-5 were classified as the residual food group. The proportion of the residual food group in the prolonged group was significantly higher than that in the normal group.

The correlations between oral phase and different oral functions are shown in Table 2. Oral phase was correlated with / pa/, / ta/, / ka/, MMSE score, RSST score, saliva flow rate, and tongue pressure. ODK elements were highly correlated with each other $(\mathrm{r}>0.9, \mathrm{p}<0.01)$, suggesting high collinearity. Age, BMI, blood albumin, number of functional teeth, and FTUs were not correlated with ODK.

Tables 3 show the results of the binomial logistic regression analysis for the associations of oral functions with oral phase prolongation after adjusting for potential covariates. The analysis revealed that the total ODK, swallowing function, and tongue pressure were associated with oral phase prolongation in model 1 (unadjusted model). However, fully adjusted analysis (model 3) revealed that only total ODK was associated with oral phase prolongation. In addition, a model adjusted for all oral function measurements was also carried out. In this model, none of the oral functions was significantly associated with the duration of oral phase [for FTU: 0.84 (0.66-1.07), $\mathrm{p}=0.152$, for RSST: $0.50(0.24-$ $1.04), \mathrm{p}=0.063$, for saliva flow: $0.52(0.24-1.17), \mathrm{p}=0.114$, for tongue pressure: $0.95(0.82-1.10), p=0.506$, and for cheek pressure: $1.05(0.88-1.25), \mathrm{p}=0.568]$.

\section{Discussion}

We investigated factors related to oral phase prolongation until the initial swallowing of solid food 
in older adults. We identified ODK as a the only oral function significantly associated with duration of oral phase. ODK is considered to be important for speech motor skills, which are coordinated by articulators such as the lips, tongue, and velum (10). Regarding ODK syllables, / pa/ is associated with lip function, / ta/ with the anterior part of the tongue, and / $\mathrm{ka} /$ with the posterior part of the tongue (16). The number of repetitions of the syllable / $\mathrm{pa} /$ has been reported to be associated with the ability to crush and mix, in terms of masticatory performance, in young dentate adults (17). The number of repetitions of the syllable / ka/ has been shown to be associated with mixing ability among complete denture wearers (18). The syllable / ta/ has been shown to significantly affect masticatory performance, as assessed by measuring the dissolved glucose emitted by gummy jelly using a glucose measuring device (19). Further, / ta/ has been shown to be significantly correlated with the ability to mix food in older adults (20). Taken together, the deterioration of ODK related lip and tongue functions is closely associated with oral phase prolongation.

Previous reports have demonstrated that ODK deteriorates at more advanced ages, and is closely associated with sarcopenia, dynapenia, physical fitness, global cognitive function, and frailty $(21,22)$. The improvement program for oral function, which includes tongue and vocal exercises, has improved ODK $(23,24)$, suggesting that such exercises might improve the duration of oral phase. As these exercises are safe and do not need any specific device, they can be easily implemented by older adults in institutions or at home.

In the present study, an unadjusted binomial logistic regression analysis (model 1) revealed that, besides ODK, swallowing function assessed by RSST and tongue pressure was associated with the duration of the oral phase. However, analyses adjusted for confounders were not statistically significant. None of saliva flow, swallowing, cheek and tongue pressure were not significantly associated with the outcome. This was partially contrary to our hypothesis. Although further investigations are needed to find out the reasons, it is possible that the absence of significant associations was related with ease of forming bolus of Processlead $\AA(14)$.

The cause of oral phase prolongation without swallowing is still unknown. A previous study reported that degradation of pharyngeal and supraglottic sensitivity progresses with increasing age (25), while another suggested that aging leads to an increased threshold of deglutition and laryngeal atresia reflexes, with the threshold volume of pharyngeal swallowing being significantly higher in older individuals (26). These findings suggest that hypoesthesia of the pharynx might cause difficulty in swallowing, leading to prolongation of the oral phase. Thus, ODK may help to squeeze the food bolus into the pharynx.

The present study had several limitations. First, because this was a cross-sectional study, the causal relation between prolongation of oral phase and ODK deterioration was not examined. Second, we used Processlead ${ }^{\circledR}$ to assess oral phase. To evaluate the actual oral phase, it might be necessary to use another type of prepared food. Third, this study was carried out at three nursing homes in a single city of Japan, potentially limiting the generalizability of the results. Fourth, the sample size of this study was small. To improve the generalizability of the results from this study, further studies with larger sample sizes are necessary. The strength of this study was that it evaluated oral functions using objective indexes including swallowing function and chewing function. Past studies have evaluated swallowing function using the EAT-10, a questionnaire survey for swallowing function $(27,28)$.

In conclusion, ODK deterioration is closely associated with oral phase prolongation until first swallowing occurs in older adults.

Funding: This study was not supported by any funding agency.

Acknowledgments: We thank three nursing homes cooperating this study.

Conflict of interest: The authors declare no conflict of interest.

Ethical Standards: The authors declare that the study procedures comply with the current ethical standards for investigation involving human participants in Japan.

Disclosure: The authors have stated explicitly that there are no conflicts of interest in connection with this article.

\section{References}

1. Iwasaki M., et al. Oral health status: relationship to nutrient and food intake among 80-year-old Japanese adults. Community Dent Oral Epidemiol, 2014 42(5):p. 441-50.

2. Ikebe K. Significance of Oral Function for Dietary Intakes in Old People. J Nutr Sci Vitaminol (Tokyo), 2015. 61 Suppl(S74-5.

3. Chia-Hui Chen C., et al. Three Nurse-administered Protocols Reduce Nutritional Decline and Frailty in Older Gastrointestinal Surgery Patients: A Cluster Randomized Trial. J Am Med Dir Assoc, 2018.

4. Fujii W., et al. Examination of chew swallow in healthy elderly persons: Does the position of the leading edge of the bolus in the pharynx change with increasing age? Jpn J Compr Rehabil Sci, 2011. 2(48-53.

5. Woda A., et al. Adaptation of healthy mastication to factors pertaining to the individual or to the food. Physiol Behav, 2006. 89(1):p. 28-35.

6. Ideno Y., et al. Evaluation of a Japanese version of the Mini-Mental State Examination in elderly persons. Geriatr Gerontol Int, 2012. 12(2):p. 310-6.

7. Persson E., et al. Repetitive Saliva Swallowing Test: Norms, Clinical Relevance and the Impact of Saliva Secretion. Dysphagia, 2018.

8. Oguchi K., et al. The Repetitive Saliva Swallowing Test (RSST) as a Screening Test of Functional Dysphagia (2) Validity of RSST. Jpn J Rehabil Med, 2000. 37(6):p. 383-388.

9. Tsuga K., et al. Manometric evaluation of oral function with a hand-held balloon probe. J Oral Rehabil, 2011. 38(9):p. 680-5.

10. Ziegler W. Task-related factors in oral motor control: speech and oral diadochokinesis in dysarthria and apraxia of speech. Brain Lang, 2002 80(3):p. 556-75

11. Smith C.H., et al. Effect of aging on stimulated salivary flow in adults. J Am Geriatr Soc, 2013. 61(5):p. 805-8.

12. N'Gom P I. and Woda A. Influence of impaired mastication on nutrition. J Prosthet Dent, 2002. 87(6):p. 667-73.

13. Nokubi T., et al. Validity and reliability of a visual scoring method for masticatory ability using test gummy jelly. Gerodontology, 2013. 30(1):p 76-82.

14. Shibata S., et al. Efficacy of a novel training food based on the process model of feeding for mastication and swallowing: A study among dysphagia patients. Jpn J Compr Rehabil Sci, 2017. 8(82-87.

15. Hiiemae K.M. and Palmer J.B. Food transport and bolus formation during complete feeding sequences on foods of different initial consistency. Dysphagia, 1999. 14(1):p. 31-42.

16. Hartelius L. and Lillvik M. Lip and tongue function differently affected in 
individuals with multiple sclerosis. Folia Phoniatr Logop, 2003. 55(1):p. 1-9.

17. Yamada A., et al. Association between tongue and lip functions and masticatory performance in young dentate adults. Journal of Oral Rehabilitation, 2015. 42(11):p. 833-839.

18. Komagamine Y., et al. Association between tongue and lip motor functions and mixing ability in complete denture wearers. Aging Clin Exp Res, 2018

19. Morita K., et al. Factors related to masticatory performance in healthy elderly individuals. J Prosthodont Res, 2018. 62(4):p. 432-435.

20. Kikutani T., et al. Oral motor function and masticatory performance in the community-dwelling elderly. Odontology, 2009. 97(1):p. 38-42.

21. Suzuki M., et al. Relationship between characteristics of skeletal muscle and oral function in community-dwelling older women. Arch Gerontol Geriatr, 2018. 79(171-175.

22. Kugimiya Y., et al. Relationship between mild cognitive decline and oral motor functions in metropolitan community-dwelling older Japanese: The Takashimadaira study. Arch Gerontol Geriatr, 2019. 81(53-58.

23. Sugiyama T., et al. Effect of swallowing exercises in independent elderly. Bull Tokyo Dent Coll, 2013. 54(2):p. 109-15.
24. Sakayori T., et al. Longitudinal Evaluation of Community Support Project to Improve Oral Function in Japanese Elderly. Bull Tokyo Dent Coll, 2016. 57(2):p. 75-82.

25. Aviv J.E., et al. Age-related changes in pharyngeal and supraglottic sensation. Ann Otol Rhinol Laryngol, 1994. 103(10):p. 749-52.

26. Shaker R., et al. Effect of aging, position, and temperature on the threshold volume triggering pharyngeal swallows. Gastroenterology, 1994. 107(2):p. 396-402.

27. Nishida T., et al. Utility of the Eating Assessment Tool-10 (EAT-10) in Evaluating Self-Reported Dysphagia Associated with Oral Frailty in Japanese Community-Dwelling Older People. J Nutr Health Aging, 2020. 24(1):p. 3-8.

28. Kilinc H.E., et al. The Effects of Different Exercise Trainings on Suprahyoid Muscle Activation, Tongue Pressure Force and Dysphagia Limit in Healthy Subjects. Dysphagia, 2019. 